\title{
Auditory detection and optimal response biases
}

\author{
ANN L. HUME* \\ University of Auckland, Auckland, New Zealand
}

\begin{abstract}
Rats were trained to detect a signal consisting of an increment in the intensity of a random noise. The procedure was analogous to the yes-no method of human psychophysics, in that one response was defined as correct and reinforced with brain stimulation if the signal was presented, and another response was correct and reinforced if the noise alone was presented. Incorrect responses produced periods of time-out. Bias functions showing how the animals' response probabilities varied as the signal intensity was reduced were obtained in two ways. In Experiment 1, the probability of presenting the signal was varied over four values between 0.4 and 0.6 . In Experiment 2, the number of brain stimulations consequent upon a correct response in the presence of the signal was varied over four values between $3: 1$ and 1:2. Differences of 0.10 and 0.05 in the signal probability, and unit differences in the ratio of brain stimulations, resulted in distinctly different bias functions. Accuracy of detection increased with the signal intensity, and was independent of the animals' response biases. When the signal probability was varied, the animals optimized the number of correct trials, and hence the number of brain stimulations obtained. When the ratio of brain stimulations was varied, the animals compromised between optimizing the number of correct trials and optimizing the number of brain stimulations obtained.
\end{abstract}

A number of studies have shown that the response biases of organisms performing in yes-no psychophysical procedures may be varied by altering such non-sensory variables as the probability of presenting a signal or the symmetry of the payoff matrix that specifies the outcomes for correct and incorrect responses. When stimulus parameters are held constant, the detectability of a signal is typically found to remain constant and independent of changes in response bias. These findings have been obtained both with humans (Tanner $\&$ Swets, 1954; Swets, Tanner, \& Birdsall, 1961; Green \& Swets, 1966; Galanter \& Holman, 1967, Markowitz \& Swets, 1967; Schulman \& Greenberg, 1970) and with animals (Stubbs, 1968; Clopton, 1972; Elsmore, 1972; Terman \& Terman, 1972; Hume \& Irwin ${ }^{1}$ ).

In experiments in which the signal probability has been a variable, it has usually been manipulated over ranges between 0.1 and 0.9 , in step sizes of 0.1 and 0.3 . These studies have shown, for both human and animal Ss, that the probability of reporting a signal is almost perfectly ranked with respect to the probability of occurrence of that signal. Such response probabilities may or may not match the signal probabilities, depending upon the value of the signal probability and the detectability of the signal (Thomas \& Legge, 1970; Terman \& Terman, 1972). When the signal detectability is reduced to zero, the psychophysical procedure becomes identical to and may be related to the probability-learning procedure.

*This research constituted a portion of a thesis submitted in partial fulfillment of the requirements for the PhD degree at the University of Auckland. The research was supported in part by grants from the New Zealand University Grants Committee and the Scientific Research Distribution Committee to R. J. Irwin. Thanks are extended to R. J. Irwin, D. M. Webster, and M. C. Davison for assistance. Reprints may be obtained from the author, Department of Psychology, University of Auckland, Private Bag, Auckland, New Zealand.
No study is known from research in either psychophysics or probability learning with humans or animals in which the effects of changes in stimulus probabilities of less than 0.10 have been investigated. This may be because many hundreds of trials of presenting a stimulus with a given probability must occur before that probability can be expected to exert a stable effect on behavior. The use of animal Ss and the maintenance of behavior by brain-stimulation reinforcement facilitates such research. In the first experiment reported here, the response probabilities of rats, performing both in an analogue of the yes-no method of human psychophysics and in a probability-learning task, were studied as a function of stimulus probabilities which varied between 0.4 and 0.6 in steps of 0.10 and 0.05 .

In psychophysical studies with humans, in which the symmetry of the payoff matrix has been a variable, Ss have usually been rewarded and punished by the use of points or money. Green and Swets (1966) and Galanter and Holman (1967), for example, have shown that when both the relative values and costs associated with the four possible response outcomes in a yes-no psychophysical procedure are varied, human Ss will rank their response probabilities systematically in relation to the different ratios of payoff. These studies have also shown that Ss will exhibit response probabilities which approximately optimize the overall outcomes of their responses under each ratio.

Psychophysical studies with animals in which the contingencies of reinforcement specifying response outcomes have been varied are also few in number. Nevin (1964) showed that changes in the probability of reinforcement, and Boneau and Cole (1967) showed that changes in deprivation would result in changes in response biases. Stubbs (1968) required pigeons to 
discriminate between stimuli of different durations, and demonstrated that their response biases were affected by asymmetric contingencies of reinforcement. Hume and Irwin, ${ }^{1}$ however, found that the response biases of rats detecting increments in the intensity of noise were unaffected by large changes in the relative duration of time-out consequent upon incorrect responses. In the second experiment reported here, response biases were studied as a function of the relative magnitude of reinforcement consequent upon correct responses, namely, the relative number of brain stimulations contingent upon these correct responses.

\section{METHOD}

\section{Subjects}

Two male albino rats, designated A-VII and A-IX, served. They had had previous extensive experience in similar experiments (Hume \& Irwin 1 ). They were given unlimited access to food and water in their home cages.

\section{Apparatus}

The experimental chamber was constructed of transparent Perspex side walls and ceiling, aluminum front and rear walls, and a grid floor. Its internal dimensions were $0.30 \mathrm{~m}$ long, $0.25 \mathrm{~m}$ wide, and $0.26 \mathrm{~m}$ high. Two stainless steel levers, requiring a force of $0.15 \mathrm{~N}$ to depress them, protruded $18 \mathrm{~mm}$ through the front wall, $165 \mathrm{~mm}$ from the floor, and $40 \mathrm{~mm}$ from each side wall. A set of lights above each of these levers indicated when a trial was in progress. A third lever, requiring a force of $0.14 \mathrm{~N}$ to depress it, was located centrally, $45 \mathrm{~mm}$ above the floor. A speaker (Rola Co., Model C) was also mounted centrally on the front wall, $190 \mathrm{~mm}$ above the floor. It was $90 \mathrm{~mm}$ in diam and was set plane with the wall.

The compartment was placed inside a ventilated sound-attenuating chamber containing a houselight. The Ss were rewarded by a burst of brain stimulation from a constant current source. It was delivered through a lead mounted in the ceiling of the chamber. Each brain stimulation consisted of a $0.5-\mathrm{sec}$ burst of a $50-\mathrm{Hz}$ alternating current, adjusted in level for each animal, and monitored by an ammeter and an oscilloscope.

The auditory stimuli consisted of $0.5-\mathrm{sec}$ bursts of random noise of undetermined bandwidth; they were generated by a Zener diode and then amplified. The frequency spectrum of the noise produced by the speaker was measured inside the open chamber approximately $40 \mathrm{~mm}$ above the center response lever at octave intervals by means of a Bruel and Kjaer sound-level meter (Type 2203) and a Bruel and Kjaer octave filter set (Type 1613). The spectrum varied $\pm 8 \mathrm{~dB}$ between center frequencies of 63 and $8,000 \mathrm{~Hz}$. On any trial, one of two stimuli differing only in intensity was presented. The intensity of one stimulus, defined as noise alone, was held constant throughout the experiment at $69 \mathrm{dBA}$, as measured on the A-weighting network of a Dawe sound-level meter (Type $1400 \mathrm{G}$ ) placed inside the open chamber. The intensity of the second stimulus, defined as the signal plus noise, was varied from 0 to $15 \mathrm{~dB}$ above the intensity of the noise alone by means of a variable attenuator (Hewlett-Packard, Type 350C). The voltage across the speaker was monitored by a quasi-RMS voltmeter (Bruel and Kjaer, Type 2410). The ambient noise level within the chamber did not exceed $35 \mathrm{dBA}$.

All experimental events were programmed and recorded automatically. The presentation of either stimulus on any trial was controlled by a specially constructed "probability generator," which contained a multivibrator free-running at $10,000 \mathrm{~Hz}$. By varying the relative time that the multivibrator was in one of two states, the probability of presenting signal plus noise could be controlled. The nominal probabilities used in the experiment were: $0.40,0.45,0.50,0.55$, and 0.60 . The corresponding actual probabilities, as based on estimates from samples of 10,000 trials, were $0.398,0.443,0.507,0.551$, and 0.619 . For convenience, the nominal probabilities are used in this paper to refer to the actual probabilities presented.

\section{Procedure}

Electrode Implantation and Histology. The animals were implanted bilaterally, under sodium pentobarbital (Nembutal) anaesthesia, with acrylic-insulated, stainless steel, monopolar electrodes. The $0.5-\mathrm{mm}$ stimulating tips were aimed at the medial forebrain bundle. The loci of the tips, in terms of the Kreig stereotaxic coordinates, were $0.88 \mathrm{~mm}$ posterior and $1.7 \mathrm{~mm}$ lateral to bregma, and $8.2 \mathrm{~mm}$ ventral to the top of the skull. An indifferent electrode was provided by a stainless steel wire loop placed over the top of the skull. The electrodes were attached to the skull with dental cement and stainless steel screws. After the experiments, the animals were sacrificed and perfused with saline and neutral-buffered Formalin solutions. The brains were washed in water, quenched in Freon and held in liquid nitrogen prior to sectioning in a cryostat microtome. Sections were placed in a photographic enlarger, and prints were made (Thompson, 1971). All electrode tips were found to be in the lateral hypothalamus.

Preliminary Training. The animals were trained to start a trial by pressing the center lever of the compartment. The panel lights were then turned on and either of two intensities of noise was presented for $0.5 \mathrm{sec}$ : noise alone at an intensity of $69 \mathrm{dBA}$ or signal plus noise at an intensity of $84 \mathrm{dBA}$. For convenience, these stimuli are referred to hereafter as the noise, $n$, and the signal, sn. A single response on the left-hand lever in the presence of the noise, $N / n$, or on the right-hand lever in the presence of the signal, $\mathrm{SN} / \mathrm{sn}$, produced a $0.5-\mathrm{sec}$ burst of brain stimulation. These events correspond to correct rejections and hits in the terminology of detection theory. The current levels were maintained at levels which had previously produced rapid responding with each $S$. The RMS values of the current were 80 microA for both animals. An incorrect response in the presence of the noise, $\mathrm{SN} / \mathrm{n}$, or in the presence of the signal, $\mathrm{N} / \mathrm{sn}$, corresponding to a false alarm and a miss, produced a $5-\mathrm{sec}$ period of time-out, during which all lights were extinguished and responses had no scheduled consequence. Incorrect trials were not repeated (a noncorrection procedure), and, after preliminary training, no additional inter- or intrastimulus responses occurred. The duration of a trial was set at $5 \mathrm{sec}$, so that if an animal did not respond on either side lever within $5 \mathrm{sec}$ of pressing the center lever, another press on the center lever was necessary to start a new trial. On average, this occurred approximately once in 5,000 trials, and the trial was not scored or included in the experimental results.

Experiment 1. The probability of presenting the signal, p(sn), was held constant at one of four values, while the signal intensity was reduced from 15 to $0 \mathrm{~dB}$ relative to the noise intensity ( $69 \mathrm{dBA})$. The relative intensities of the signal were $15,10,8,5$, $3,2,1$, and $0 \mathrm{~dB}$. As in the preliminary training, a hit or a correct rejection produced a 0.5 -sec burst of brain stimulation, and a false alarm or a miss produced a 5 -sec period of time-out. For each series of signal intensities, one animal, A-VII, was exposed to signal probabilities of $0.4,0.6,0.5$, and 0.45 , in that order. The other animal, A-IX, was exposed to signal probabilities of $0.6,0.4,0.5$, and 0.55 . Animals were exposed to each signal intensity at each signal probability for three successive daily sessions. Each session lasted approximately $1 \mathrm{~h}$ and consisted of 1,000 test trials preceded by 200 warm-up trials. Data were recorded in blocks of 500 trials.

Experiment 2. The number of bursts of brain stimulation consequent upon a correct rejection, $\mathrm{N} / \mathrm{n}$, relative to the number of bursts consequent upon a hit, $\mathrm{SN} / \mathrm{sn}$, was held constant at one of three values, while the signal intensity was reduced from 15 to $0 \mathrm{~dB}$ above the noise intensity. For each ratio of brain stimulation, the series of signal intensities presented was the 
Fig. 1. Bias functions obtained with a series of decreasing signal intensities at each of the signal probabilities indicated. The probability of a hit is represented by the probability of a response on the right-hand lever in the presence of the signal, $\mathrm{p}(\mathrm{SN} \mid \mathrm{sn})$; the probability of a false alarm is represented by the probability of a response on the right-hand lever in the presence of the noise, $\mathrm{p}(\mathrm{SN} \mid \mathrm{n})$. Each point is based upon 2,000 trials.
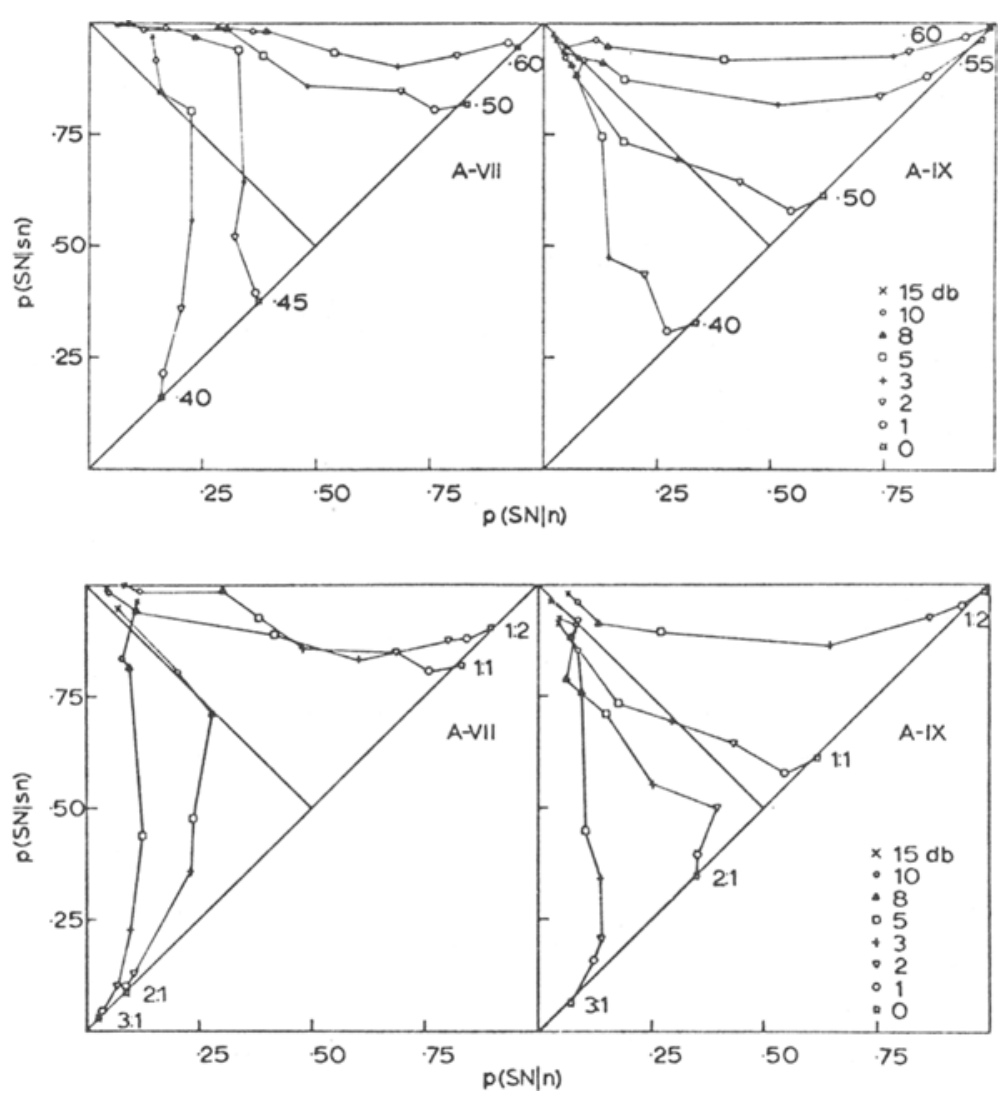

Fig. 2. Bias functions obtained with a series of decreasing signal intensities at each of the ratios of brain stimulation for correct rejections to hits indicated. The signal probability was 0.5 . The bias function obtained for each $S$ when the signal probability was 0.5 and the ratio of brain stimulations was $1: 1$ is reproduced from Fig. 1. Each point is based upon 2,000 trials.

same as that in Experiment 1. When more than one burst of brain stimulation was presented for a correct response, the duration of each burst was $0.5 \mathrm{sec}$ and the interval between bursts was $0.5 \mathrm{sec}$. The probability of presenting the signal was held constant at 0.5 , and both a miss and a false alarm produced a 5 -sec period of time-out. One animal, A-VII, was exposed to ratios of brain stimulation for correct rejections to hits of $2: 1$, $3: 1$, and $1: 2$, in that order. The other animal, $A-I X$, was exposed to ratios of $1: 2,2: 1$, and $3: 1$.

Before each new ratio of brain stimulations was introduced, the animals were given two sessions of training at a signal intensity of $15 \mathrm{~dB}$, during which correct rejections and hits both produced single bursts of brain stimulation. As in Experiment 1, animals were exposed to each signal intensity at each ratio of brain stimulation for three successive daily sessions.

\section{RESULTS}

Figures 1 and 2 show how the probability of a hit, $\mathrm{p}(\mathrm{SN} \mid \mathrm{sn})$, covaried with the probability of a false alarm, $\mathrm{p}(\mathrm{SN} \mid \mathrm{n})$, at each signal intensity in Experiments 1 and 2. The lines in Fig. 1 connect points obtained at the same signal probability, and those in Fig. 2 connect points obtained at the same ratio of brain stimulations for correct rejections to hits. These lines, within the receiver-operating-characteristic (ROC) space of signal detection theory (Green \& Swets, 1966), thus represent bias functions. Each point on a function was obtained at a different signal intensity and is based upon 2,000 trials.

The levels of detection exhibited by the animals for each signal intensity under the different signal probabilities and ratios of brain stimulation may be described by the bias-free index of detectability, $\mathrm{d}^{\prime}$ (Green \& Swets, 1966). Figures 3 and 4 show values of $\mathrm{d}^{\prime}$ plotted as a function of the relative intensity of the signal. The functions for A.VII have been displaced by one $\log$ unit on the abscissae. The parameter of each function is either the signal probability, $\mathrm{p}(\mathrm{sn})$ (Fig. 3), or the ratio of brain stimulations for correct responses, B.S. (Fig. 4). Each value of $\mathrm{d}^{\prime}$ is based upon the conditional probabilities of response represented by each point in Figs. 1 and 2 (cf. Elliott, 1964).

In these figures, $\mathrm{d}^{\prime}$ increases from 0.0 when the signal intensity was $0 \mathrm{~dB}$ to values exceeding 3.0 when the signal intensity was $15 \mathrm{~dB}$. For A-IX, this relationship was independent of changes in both the signal probability and the ratio of brain stimulations. In contrast, A-VII exhibited slightly lower levels of detection when the signal probability was 0.4 and 0.6 , and when the ratio of brain stimulations was $2: 1$ and 3:1.

Although the variance associated with $\mathrm{d}^{\prime}$ cannot be calculated, the variation in the individual values of $\mathrm{d}^{\prime}$ at each signal intensity in Figs. 3 and 4 is generally small, particularly at low signal intensities. This result indicates that detection remained constant and independent of changes in the signal probability and the ratio of brain stimulations. It also means that levels of detection were maintained at each signal intensity, even though a lower level of detection could have achieved the same or a greater number of brain stimulations when either the 

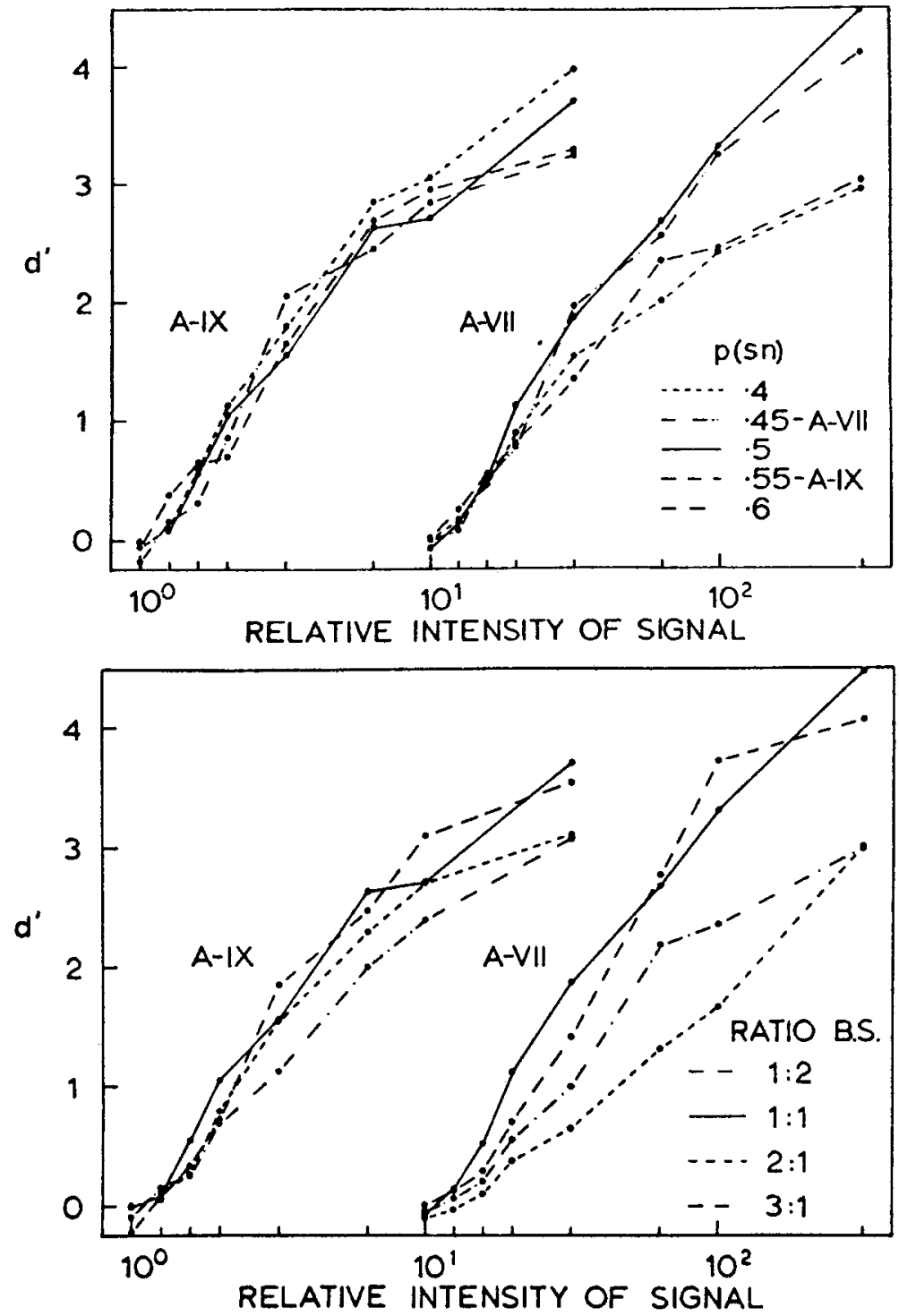

Fig. 3. The index of detectability, $d^{\prime}$, as a function of the relative intensity of the signal at each signal probability, p(sn). Each value of $d^{\prime}$ is based upon the conditional probabilities of response represented by each point in Fig. 1. The functions for A-VII have been displaced by one log unit on the abscissa.
Fig. 4. The index of detectability, $d^{\prime}$, as a function of the relative intensity of the signal at each ratio of brain stimulations for correct rejections to hits, B.S. Each value of d' is based upon the conditional probabilities of response represented by each point in Fig. 2. The functions for A-VII have been displaced by one log unit on the abscissa. signal probability or the ratio of brain stimulations was varied, relative to the level of detection necessary at that signal intensity to achieve a given number of brain stimulations when the signal probability was 0.5 and the ratio of brain stimulations was $1: 1$.

In Figs. 1 and 2, no bias for one response over the other is represented by points which lie along the minor diagonal; this diagonal represents equal probabilities of response to the two levers. Relative bias to respond to the right-hand lever is proportional to the angular displacement of a point above the minor diagonal, and relative bias to respond on the left-hand lever is proportional to the angular displacement of a point below that diagronal. These biases are analogous to biases toward reporting "yes" or "no" in human detection settings. The bias functions in Figs. 1 and 2 show that the animals exhibited varying degrees of bias to one or the other lever as a joint function of the signal intensity and either the signal probability or the ratio of brain stimulations for correct responses.

When the signal probability was 0.5 and the ratio of brain stimulations was $1: 1$, the bias function obtained for A-IX lies close to the minor diagonal and the angular departures of the points from this line do not represent a consistent bias to either lever. The same bias function for A-VII, in contrast, represents a strong and consistent preference to respond on the right-hand lever despite the symmetrical payoff and equal stimulus probabilities. Such biases are frequently observed in two-choice psychophysical procedures with animals (e.g., Irwin \& Terman, 1970; Blough, 1971; Hodos \& Bonbright, 1972).

At large signal intensities, the points on all the bias functions for A-IX lie on or very close to the minor diagonal of Figs. 1 and 2. Corresponding points for A-VII lie slightly above the minor diagonal. Thus, at large signal intensities, response biases were relatively independent of variations in the signal probability 
Fig. 5. Theoretical bias functions. The straight lines represent the relationship between $p(S N \mid s n)$ and $p(S N \mid n)$ that would result as a function of signal intensity if a $S$ matched his probability of response on the right-hand lever, $p(\mathrm{SN})$, to the signal probability when the payoff matrix is fixed and symmetrical, or to the ratio of the number of brain stimulations available for correct responses on that lever to the number of brain stimulations available for correct responses on that lever to the number of brain stimulations available for correct responses on the left-hand lever when the signal probability is 0.5 . The parameter of each line is either the signal probability or the ratio of brain stimulations for correct rejections to hits. The curved lines represent the relationship between $p(\mathbf{S N} \mid \mathbf{s n})$ and $p(\mathbf{S N} \mid \mathbf{n})$ that would result as a function of signal intensity if a $S$ distributed his responses so as to obtain the optimal number of brain stimulations under each signal probability or ratio of brain stimulations. The parameter of each curved line, $\beta$, represents the ratio of the noise probability to the signal probability when the payoff matrix is fixed and symmetrical, or the ratio of brain stimulations for correct rejections to hits when the signal probability is 0.5 .

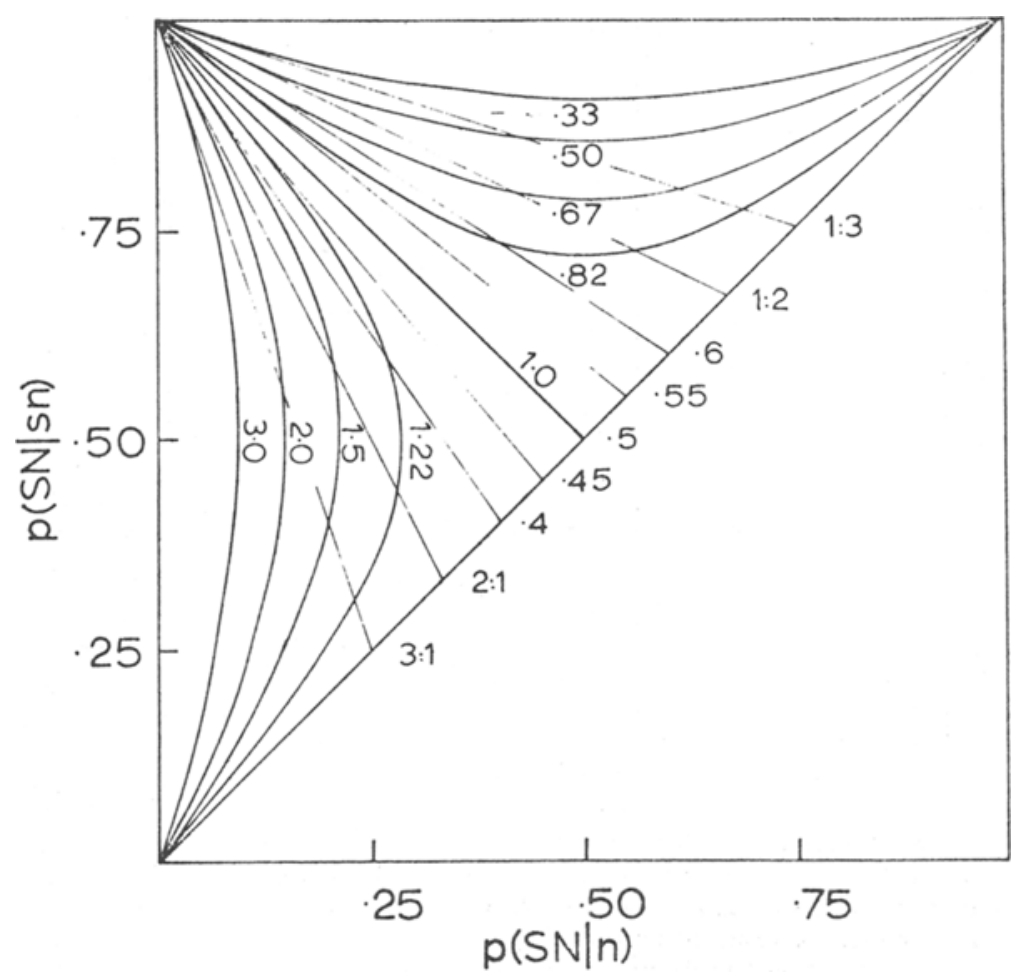

between 0.4 and 0.6 and in the ratio of brain stimulations for correct rejections to hits between $3: 1$ and $1: 2$.

Below a signal intensity of about $8 \mathrm{~dB}$, the bias functions begin to diverge from the minor diagonal and from each other, at a rate dependent on the signal probability or the ratio of brain stimulations. Differences in the signal probability of 0.10 and as small as 0.05 , and in the ratio of brain stimulations of 1.0 and 2.0 , produced fine and systematic differences in the conditional probabilities of response at each signal intensity. Differences of equal magnitude in the signal probability or the ratio of brain stimulations did not always, however, produce differences of equal magnitude in response bias. For example, the angular displacements of the bias functions obtained with A-VII when the signal probability was 0.4 and 0.6 from the bias function obtained when the signal probability was 0.5 are not equal. Similar asymmetry is apparent between the bias functions obtained with A-VII with ratios of brain stimulation of $2: 1,1: 1$, and $1: 2$. Such differences may be due to the order in which the functions were obtained, although they were of much less magnitude with A-IX.

The particular shape and loci of each function in Figs. 1 and 2 also reflect the way in which the animals changed their response biases as a joint function of the signal intensity and either the signal probability or the ratio of brain stimulations. Figure 5 shows two different sets of theoretical bias functions, which could be obtained under different signal probabilities or ratios of payoff for correct responses. The straight lines represent the loci of probabilities of hits and false alarms that would result as a function of signal intensity if a $S$ matched its probability of response to the right-hand lever, $\mathrm{p}(\mathrm{SN})$, to the signal probability when the payoff matrix is fixed and symmetrical, or to the ratio of the number of brain stimulations available for correct responses on that lever to the number of brain stimulations available for correct responses on the left-hand lever when the signal probability is 0.5 . These straight lines represent a strategy which corresponds to maintaining a constant bias to the two levers, hence isobias, as the signal intensity is reduced (cf. Linker, Moore, \& Galanter, 1964; Parks, 1966; Irwin \& Terman, 1970; Thomas \& Legge, 1970). The parameter of these functions is the signal probability when the payoff matrix is symmetrical, and the ratio of brain stimulations for correct rejections to hits when the signal probability is 0.5 .

The curved lines in Fig. 5 represent the loci of probabilities of hits and false alarms that would result as a function of signal intensity if a $S$ distributed its responses so as to obtain the maximum number of brain stimulations under each signal probability or ratio of brain stimulations. The parameter, $\beta$, of each curved line represents the ratio of the noise probability to the signal probability when the payoff matrix is fixed and symmetrical, or the ratio of brain stimulations for correct rejections to hits when both types of incorrect responses result in equal periods of time-out and the signal probability is 0.5 . A separate study would be 


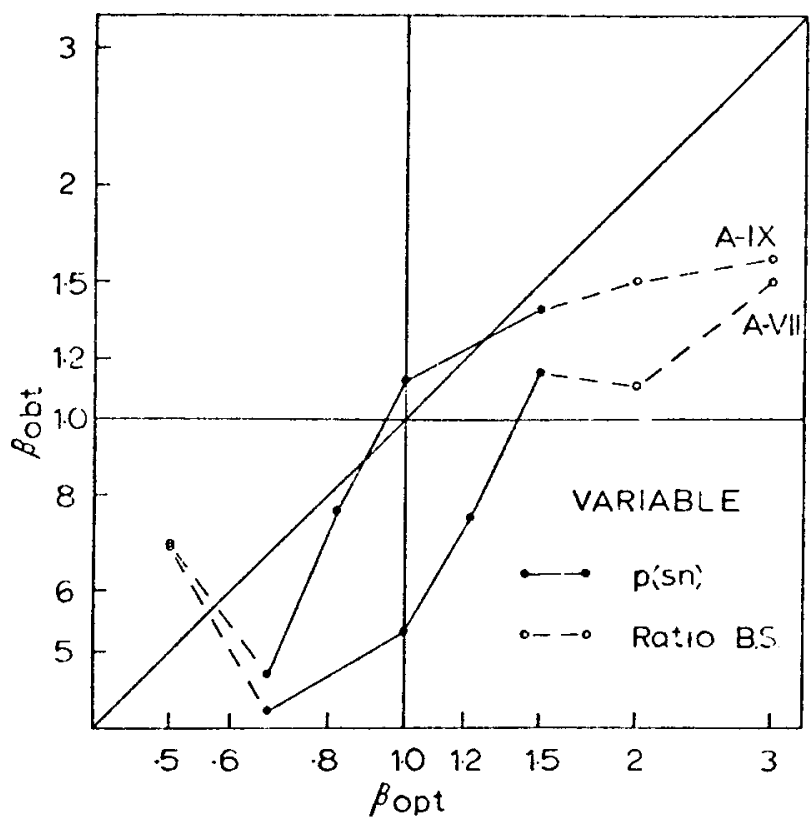

Fig. 6. Relationships between obtained response criteria $\left(\beta_{\text {obt }}\right)$ and the response criteria $\left(\beta_{\text {opt }}\right)$ which would result in the optimal number of brain stimulations (filled points) or ratio of brain stimulations (unfilled points). The values of $\beta_{\text {obt }}$ are based upon the conditional probabilities of response shown for each signal intensity between 1 and $8 \mathrm{~dB}$ in Figs. 1 and 2. The coordinates are logarithmically spaced and the major diagonal represents perfect correspondence between obtained and optimal criteria.

required to determine the relative reinforcing values of more than one brain stimulation of the parameters used in this experiment, so that an estimate of $\beta$ must assume that, for example, two brain stimulations have exactly twice the value of one, and that the effects of closely successive brain stimulation are independent. Under each signal probability, the strategy represented by each curved line corresponds to optimizing expected value, in the sense defined by statistical decision theory (cf. Wald, 1950; Luce, 1969, p. 65; Green \& Swets, 1966, pp. 21-23), and hence to optimizing the expected percentage of correct responses. Under each ratio of brain stimulations, such strategies correspond to optimizing the number of brain stimulations; they do not correspond to optimizing the expected percentages of correct responses which decrease with increasing response bias when the signal probability is 0.5 , nor do they correspond to optimizing expected value, since their determination does not take into account the costs of incorrect responses in terms of time-out. The two sets of functions shown in Fig. 5 thus represent response strategies which optimize the probability or numbers of brain stimulations (curved lines) or match the signal probability or the relative numbers of brain stimulations (straight lines) in this type of experiment. The bias functions based on these two strategies are identical only when the signal probability is 0.5 and the payoff matrix is fixed and symmetrical; in both cases, each is defined by the minor diagonal in the theoretical figure.
Comparison of the bias functions in Figs. 1 and 2 with the functions in Fig. 5 shows that the obtained functions lie closer to and more nearly resemble the theoretical optimizing functions than the theoretical matching functions. Only two bias functions for A-IX approximate a linear form and represent matching: that obtained when the signal probability was 0.4 and that obtained when the ratio of brain stimulations was $2: 1$. Further, under this signal probability and under this ratio of brain stimulations, the animal did not respond exclusively to the left-hand lever when the signal intensity was $0 \mathrm{~dB}$. If it had, it could have obtained 80 more brain stimulations in every 1,000 trials when the signal probability was 0.4 , and 360 more brain stimulations in every 1,000 trials when the ratio of brain stimulations was $2: 1$. The results in these two instances may be attributable to the order in which the bias functions were generated.

The curvilinearity of the remainder of the functions in Figs. 1 and 2, and the way in which the probabilities of hits and false alarms approach the limiting values of 0,0 or 1,1 , as the signal intensity was reduced to $0 \mathrm{~dB}$, indicate that the Ss adjusted their response biases under each signal probability or ratio of brain stimulation in such a way as to obtain the maximum number of brain stimulations. They did not exhibit matching strategies, nor did they show a constant degree of bias to one or the other lever, or isobias.

Quantitative assessment of the extent to which the animals exhibited optimal biases is provided by comparing the parameters of the curves in Fig. 5 and corresponding parameters of the curves in Figs. 1 and 2, namely, the optimal and the obtained values of the index, $\beta$. The optimal values of $\beta, \beta_{\text {opt }}$, represent the slope of the ROC curves which would describe detection along each bias function in Fig. 5. The obtained values of $\beta, \beta_{\mathbf{o b t}}$, correspondingly represent the slope of the ROC curves which would describe detection at each point on the bias functions in Figs. 1 and 2 (cf. Luce, 1959 , p. 65).

Figure 6 shows the relationship between the obtained and optimal values of $\beta$ for these experiments. The obtained values of $\beta$ represent the average slope of the ROC curves describing detection on each of the bias functions in Figs. 1 and 2 for signal intensities between 1 and $8 \mathrm{~dB}$. Points obtained when the signal intensity was 10 and $15 \mathrm{~dB}$ are omitted from this analysis since the animals' conditional probabilities of response at these levels of detection were virtually unaffected by the different signal probabilities or ratios of brain stimulation.

In Fig. 6, the filled points are based on bias functions obtained under different signal probabilities, and the unfilled points are based on functions obtained under different ratios of brain stimulation. The coordinates are logarithmically spaced, and the major diagonal represents perfect correspondence between the obtained and optimal values of $\beta$. The central horizontal axis, for 
which $\beta_{\text {obt }}=1.0$, corresponds to points lying on the minor diagonal of the ROC space, and hence to no response bias. Points lying above the central axis represent a bias to the left-hand lever, and points below, a bias to the right-hand lever. Points lying between the major diagonal and the central axis in either the upper right or lower left quadrants of the figure represent less extreme than optimal biases to the corresponding levers.

The values of $\beta_{o b t}$ for the bias functions generated when the signal probability was varied show a rank order coefficient of correlation of unity with the values of $\beta_{\text {opt }}$ for both Ss. The values of $\beta_{\text {obt }}$ for A-IX almost perfectly correspond to the optimal values, indicating that at each signal probability this $\mathrm{S}$ obtained almost the maximum number of brain stimulations for the range of signal intensities between 1 and $8 \mathrm{~dB}$. The consistent bias which A-VII showed to the right-hand lever, as represented by the displacement of the points in Fig. 6 below the major diagonal, resulted in less than a maximum number of brain stimulations being obtained under each signal probability.

The values of $\beta_{\mathrm{obt}}$ for the bias functions generated when the ratio of brain stimulations was varied show a rank order coefficient of correlation of unity with the values of $\beta_{\text {opt }}$ for A-IX. The value obtained with A-VII when the ratio was $1: 1$ deviates from perfect correlation. Correspondence between the obtained and optimal values of $\beta$ is not, however, so close as that resulting when the signal probability was varied. The functions of $\beta_{\text {ob } t}$ for both Ss are flatter.

Different ratios of brain stimulations resulted in response biases for which the obtained values of $\beta$ lay between the optimal values and 1.0. Different signal probabilities did not produce similarly consistent relationships between the obtained and optimal values of $\beta$ either in this study or in a previous study ${ }^{1}$ in which the noise and signal probabilities were varied over a wider range of ratios. Different ratios of brain stimulation thus produced less extreme response biases than did equivalent ratios of noise and signal probabilities.

\section{DISCUSSION}

In these experiments with animals, the detectability of signals consisting of increments between 0 and $15 \mathrm{~dB}$ in the intensity of random noise increased monotonically with signal intensity. Detection, in terms of the index $\mathrm{d}^{\prime}$, was independent of variations in response bias. Response bias, in turn, varied systematically with changes in either the signal probability or the ratio of brain stimulations for correct responses.

Functions relating $\mathrm{d}^{\prime}$ and signal intensity (Figs. 3 and 4) showed that detection remained virtually invariant across different signal probabilities or ratios of brain stimulation, even when the signal intensity was 1 or $2 \mathrm{~dB}$. At these signal intensities, numbers of correct trials approached chance levels, so that when the signal probability was not 0.5 , or the ratio of brain stimulation was not unity, the animals, by responding on only one lever, irrespective of the signal intensity, could have obtained as many as, or more than, the number of brain stimulations they could have obtained by detecting the same signal when the signal probability was 0.5 and the ratio of brain stimulations was $1: 1$. Figures 1 and 2 show that the animals did not exhibit exclusive biases to one or the other lever at near-zero signal intensities. Instead, they distributed their responses to the two levers as a function of either the signal probability or the ratio of brain stimulations and the signal intensity.

Variations in the signal probability of 0.10 and 0.05 from a probability of 0.5 resulted in distinct and systematic differences in the animals' response biases across the range of signal intensities presented. Variations in the ratio of brain stimulations for correct responses of 1.0 and 2.0 from a ratio of $1: 1$ resulted in similarly distinct and systematic differences in response biases. As discussed in the introduction, no other study is known in which the effects of differences of 0.05 in event probabilities have been investigated. The effects of varying relative magnitudes of reinforcement in free-operant studies with animals has been widely studied with both food (Baum \& Rachlin, 1969) and brain stimulation reinforcers (Pliskoff \& Hawkins, 1967), but such effects have not been previously investigated in a psychophysical procedure.

Under each signal probability or ratio of brain stimulations, the animals responded to obtain approximately the maximum number of brain stimulations at each signal intensity. On only two occasions did the bias functions obtained with signal probabilities other than 0.5 and nonunit ratios of brain stimulations represent matching between the response probabilities and the stimulus probabilities, in one case, and the ratio of brain stimulations, in the other. Correspondence between the response biases of the animals, indexed by $\beta_{o b t}$, and those which a $S$ would exhibit if it were optimizing the number of brain stimulations under each signal probability or ratio of brain stimulation, indexed by $\beta_{\mathrm{opt}}$, is close (Fig. 6). Such correspondence, however, in both this and in a previous study, in which the signal probability was varied between 0.1 and 0.9 , is closer under different signal probabilities than under different ratios of brain stimulation. In Fig. 6 , the values of $\beta_{\text {obt }}$ under each signal probability approximate the optimal values of $\beta$, while the values of $\beta_{o b t}$ under each nonunit ratio of brain stimulations lie consistently between the optimal values and 1.0 . Thus, in the present procedure, animals will closely optimize the total number of brain stimulations obtained when the signal probability is varied, but they will compromise between optimizing the number of brain stimulations (for which $\beta_{\mathrm{obt}}=$ $\beta_{\text {opt }}$ ) and optimizing the number of correct trials (for which $\beta_{o b t}=1.0$ ) when the relative number of brain 
stimulations for correct responses is varied.

When the signal intensity was reduced to $0 \mathrm{~dB}$, the procedure became analogous to that of probability learning. When the signal probability was 0.5 and the ratio of brain stimulations was $1: 1$, the animals distributed their responses between the two levers. Bitterman (1965) has obtained similar results with rats in a two-choice probability-learning situation. When the signal probability was not 0.5 or the ratio of brain stimulations was not unity, the animals' response probabilities to one or the other lever approached extreme values of 1.0 or 0.0 , with three exceptions (Figs. 1 and 2). The general tendency towards responding which maximized either the probability or the number of reinforcements when the signal intensity was $0 \mathrm{~dB}$ also agrees with the findings of Bitterman and his associates (Bitterman, Wodinsky, \& Candland, 1958; Bitterman, 1965) that rats will maximize the probability of reinforcement in either spatial or visual probability-learning procedures when the probability of reinforcement for one response exceeds 0.5. Shimp (1966) has similarly demonstrated maximizing in a probability-learning task with pigeons. Humans in probability-learning situations, in contrast, typically match their response probabilities to the event probabilities (Estes, 1962; Weir, 1964; Jones, 1971). They exhibit similar tendencies in psychophysical procedures when the signal probability is varied (Thomas \& Legge, 1970), although they tend to exhibit more optimal response criteria when the symmetry of the payoff matrix is varied (Green \& Swets, 1966, p. 90; Galanter \& Holman, 1967). In both types of task, therefore, the performances of animals and human Ss differ markedly. In probability-learning procedures, rats exhibit a mathematically optimal strategy of always choosing the more frequent event (cf. Flood, 1964); humans do not. In the yes-no psychophysical procedure, when the signal probability is varied, rats exhibit response biases that more closely approach statistically optimal biases as a function of signal strength than do humans. When the payoff symmetry is varied, other factors complicate comparison between the species. On the basis of the present results and the data reported by Green and Swets (1966, p. 90), however, it appears that in such cases humans may perform more optimally in a statistical sense than animals.

The effects of varying the signal probability or the ratio of brain stimulations on the animals' response biases in these experiments may also be compared with the effects of varying the relative durations of time-out consequent upon incorrect responses in an identical procedure (Hume \& $\operatorname{Irwin}^{1}$ ). Variations from 1:30 sec to $30: 1 \mathrm{sec}$ in the relative durations of time-out for the incorrect responses, false alarms and misses, produced no changes in the animals' response biases. The animals responded so as to optimize the number of correct trials, and hence the number of brain stimulations obtained, irrespective of the total time spent in time-out. They did not respond so as to optimize either the relative or the absolute rate of reinforcement per unit time.

Comparison of these results with those of the present study indicates that in this discrete-trial psychophysical procedure using brain stimulation as a reinforcer, the signal probability controls rats' response biases more effectively than the relative number of brain stimulations for correct responses, which in turn controls response biases more effectively than the relative duration of time-out for incorrect responses. The animals responded to optimize the probability of reinforcement per response before they responded to optimize the overall number of reinforcements and, in turn, before they responded to minimize the time spent in time-out, and, by implication, therefore, before they responded to optimize the overall rate of reinforcement in time.

\section{REFERENCES}

Baum, W. H., \& Rachlin, H. C. Choice as time allocation. Journal of the Experimental Analysis of Behavior, 1969, 12, 861-874.

Bitterman, M. E. Phyletic differences in learning. American Psychologist, 1965, 20, 396-410.

Bitterman, M. E., Wodinsky, J., \& Candland, D. K. Some comparative psychology. American Journal of Psychology, $1958,71,94-110$.

Blough, P. M. The visual acuity of the pigeon for distant targets. Journal of the Experimental Analysis of Behavior, 1971, 15, 57-67.

Boneau, C. A., \& Cole, J. L. Decision theory, the pigeon, and the psychophysical function. Psychological Review, 1967, 74, 123-135.

Clopton, B. M. Detection of increments in noise intensity by monkeys. Journal of the Experimental Analysis of Behavior, $1972,17,473-481$.

Elliott, P. B. Tables of d'. Appendix I in J. A. Swets (Ed.), Signal detection and recognition by human observers: Contemporary readings. New York: Wiley, 1964.

Elsmore, T. F. Duration discrimination: Effects of probability of stimulus presentation. Journal of the Experimental Analysis of Behavior, 1972, 18, 465-469.

Estes, W. K. Learning theory. Annual Review of Psychology, $1962,13,107-144$.

Flood, M. M. Environmental non-stationarity in a sequential decision-making experiment. In R. M. Thrall, C. H. Coombs, and R. L. Davies (Eds.), Decision processes. New York: Wiley, 1954.

Galanter, E., \& Holman, G. L. Some invariances of the isosensitivity function and their implications for the utility function of money. Journal of Experimental Psychology, $1967,73,333-339$.

Green, D. M., \& Swets, J. A. Signal detection theory and psychophysics. New York: Wiley, 1966.

Hodos, W., \& Bonbright, J. C., Jr. The detection of visual intensity differences by pigeons. Journal of the Experimental Analysis of Behavior, $1972,18,471-479$.

Irwin, R. J., \& Terman, M. Detection of brief tones in noise by rats. Journal of the Experimental Analysis of Behavior, 1970, 13, 135-143.

Jones, M. R. From probability learning to sequential processing: A critical review. Psychological Bulletin, 1971, 76, 153-185.

Linker, E., Moore, M. E., \& Galanter, E. Taste thresholds detection models and disparate results. Journal of Experim ental Psychology, 1964, 67, 59-66.

Luce, R. D. Individual choice behavior. New York: Wiley, 1959.

Markowitz, J., \& Swets, J. A. Factors affecting the slope of empirical ROC curves: Comparison of binary and rating responses. Perception \& Psychophysics, 1967, 2, 91-100.

Nevin, J. A. A method for the determination of psychophysical functions in the rat. Journal of the Experimental Analysis of Behavior, 1964, 7, 169(A).

Parks, T. E. Signal-detectability theory of recognition memory perform ance. Psychological Review, 1966, 73, 44-58.

Plisk off, S. S., \& Hawkins, T. D. A method for increasing the reinforcement magnitude of intracranial reinforcement. Journal of the Experimental Analysis of Behavior, 1967, 10, 281-289. 
SchuIman, A. I., \& Greenberg, G. Z. Operating characteristics and a priori probability of the signal. Perception \& Psychophysics, 1970, 8, 317-320.

Shimp, C. P. Probabilistically reinforced behavior in pigeons. Journal of the Experimental Analysis of Behavior, 1966, 9 , 433-455.

Stubbs, A. The discrimination of stimulus duration by pigeons. Journal of the Experimental Analysis of Behavior, 1968, 11, 223-238.

Swets, J. A., Tanner, W. P., \& Birdsall, T. G. Decision processes in perception. Psychological Review, 1961, 68, 301-340.

Tanner, W. P., \& Swets, J. A. A decision-making theory of visual detection. Psychological Review, 1954, 61, 401-409.

Terman, M., \& Terman, J. S. Concurrent variation of response bias and sensitivity in an operant-psychophysical test. Perception \& Psychophysics, 1972, 11, 428-432.

Thom as, E. A. C., \& Legge, D. Probability matching as a basis for detection and recognition decisions. Psychological Review, 1970, 77, 65-72.
Thompson, R. Introducing subcortical lesions by electrolytic methods. In R. D. Myers (Ed.), Methods in psychobiology. Vol. 1. London: Academic Press, 1971. Pp. 131-153.

Wald, A. Statistical decision functions. New York: Wiley, 1950.

Weir, M. W. Developmental changes in problem-solving strategies. Psychological Review, 1964, 71, 473-490.

\section{NOTE}

1. Hume, A. L., and Irwin, R. J. Bias functions and operating characteristics of rate discriminating auditory stimuli. In preparation.

(Received for publication June 18, 1973; revision received September 19, 1973.) 